ÉGYPTE monde arabe

\section{Égypte/Monde arabe}

12-13 | 1993

Une économie en transition

\title{
Quelques conséquences sociales des programmes d'ajustement structurel
}

Heba Ahmed Nassar

\section{OpenEdition}

\section{Journals}

Édition électronique

URL : https://journals.openedition.org/ema/1262

DOI : 10.4000/ema.1262

ISSN : 2090-7273

\section{Éditeur}

CEDEJ - Centre d'études et de documentation économiques juridiques et sociales

\section{Édition imprimée}

Date de publication : 31 mars 1993

Pagination : 145-177

ISSN : 1110-5097

\section{Référence électronique}

Heba Ahmed Nassar, «Quelques conséquences sociales des programmes d'ajustement structurel», Égypte/Monde arabe [En ligne], 12-13 | 1993, mis en ligne le 08 juillet 2008, consulté le 07 juillet 2022. URL : http://journals.openedition.org/ema/1262 ; DOI : https://doi.org/10.4000/ema.1262

Ce document a été généré automatiquement le 7 juillet 2022.

Tous droits réservés 


\section{Quelques conséquences sociales des programmes d'ajustement structurel}

Heba Ahmed Nassar

1 Cet article n'a pas pour objectif de critiquer les programmes d'ajustement structurel ni d'en évaluer l'efficacité. Nous essaierons plutôt, dans ces pages, de mettre en évidence certaines de leurs conséquences sociales ${ }^{1}$, ce qui suppose de définir les divers domaines qu'ils couvrent et de comprendre clairement leur interaction à la lumière des spécificités de chaque domaine. À cet effet, nous classerons les politiques d'ajustement en trois catégories, chacune pouvant être associée à un impact donné sur le bien-être social.

2 a) La contraction de la demande intérieure de produits importés et locaux doit permettre de diminuer les importations et d'accroître les exportations. Elle nécessite une réduction des dépenses publiques par la suppression des aides et des subventions directes, une politique salariale visant à la baisse des salaires réels ainsi que le relèvement des taux d'intérêt et le plafonnement du crédit, stimulant l'épargne locale et stabilisant l'offre de monnaie. Elle exige également l'unification des taux de change, qui élimine les subventions implicites aux produits bénéficiant du cours de la livre le plus élevé et les distorsions dans l'allocation intérieure des ressources entre les produits. Enfin, elle requiert d'augmenter les taxes indirectes pour accroître les revenus de l'État tout en réduisant la demande intérieure.

3 Pour évaluer les conséquences sociales de ces mesures, il convient de déterminer le niveau de la demande globale : s'il est inférieur à celui de l'offre qui assurerait le pleinemploi, la politique d'ajustement permettra peut-être, à court terme, de rééquilibrer la balance extérieure tout en réduisant le niveau de l'emploi. Dans l'hypothèse où la demande globale de biens exportables ne diminuerait pas grâce à la hausse des exportations, la demande intérieure de biens non exportables, tels que les services et la construction, baisserait probablement, entraînant une réduction du taux d'activité et des revenus dans ces secteurs. 
4 b) Il s'agit ensuite - à travers l'affectation des ressources économiques, travail et capital - de réorienter les dépenses publiques du secteur des services vers le secteur productif des produits exportables, et de la consommation vers l'investissement.

Cela suppose de libérer les prix et de modifier les prix relatifs des produits au profit des biens exportables, en affectant les ressources à l'exportation ou aux productions locales destinées à remplacer les importations. Ces politiques permettraient, en fin de compte, de compenser la baisse de production dans le secteur des biens non exportables par son augmentation dans celui des biens exportables. Insistons à cet égard sur le fait que le succès de cette politique exige une grande souplesse dans la gestion des ressources, elle-même liée aux techniques de production utilisées, au degré de compétence de la main-d'œuvre et à sa capacité d'adaptation aux processus de conversion.

6 On trouve encore dans cette catégorie des mesures touchant à l'emploi, d'autres relatives au commerce extérieur - visant à libérer les taux de change, à encourager les exportations et à limiter les importations - à la fixation des prix et à la révision des prix de l'énergie.

7 c) Dernière catégorie de mesures : mettre en place des réformes structurelles telles que la libéralisation du commerce, la limitation du rôle de l'État, la réforme des marchés financiers, la diminution des contrôles des changes, la réforme des prix... Mesures qui visent à encourager l'économie de marché et l'intégration au monde extérieur, à augmenter la croissance et l'efficacité économique par le développement du système des incitations, enfin à améliorer la capacité de la production à répondre à la conjoncture.

\section{Cadre général de l'étude}

\section{Les déterminants de l'étude}

Avant de présenter la problématique générale, il importe de mettre en évidence un ensemble de facteurs, certains liés à la nature même des programmes d'ajustement structurel, d'autres à leurs effets sociaux.

\section{Facteurs liés à la nature des programmes d'ajustement structurel}

On remarque, pour commencer, de nettes divergences dans les effets prévisibles des divers programmes d'ajustement. Ainsi peut-on s'attendre à ce que la diminution des dépenses publiques ait des effets déflationnistes du fait de la limitation de l'offre de monnaie, de la baisse des dépenses gouvernementales et de la croissance des salaires, avec les conséquences négatives qui s'ensuivent sur les revenus et le chômage. Quant aux politiques de réorientation des dépenses publiques du secteur des services vers le secteur productif et de la consommation vers l'investissement, elles favorisent par nature une expansion économique, puisqu'elles transfèrent les ressources productives vers les secteurs de biens exportables. De même, l'amélioration de l'efficacité économique par le développement du système des incitations et l'ajustement de la production aux indicateurs du marché visent la croissance à long terme, et leurs effets sociaux n'ont pas encore été évalués. 
De la remarque précédente résulte une interaction temporaire entre les effets des programmes d'ajustement structurel et des effets déflationnistes soudains ${ }^{2}$; d'où la nécessité de mettre en place des programmes à court terme pour compenser :

1. leurs conséquences négatives sur le niveau de l'emploi ;

2. les effets inflationnistes à moyen terme des politiques de transfert des ressources vers les secteurs d'exportation, du fait de l'importance des ressources financières nécessaires à leur application, de la rigidité de l'emploi et du faible niveau de qualification de la maind'œuvre ;

3. le temps nécessaire à la réalisation des réformes structurelles, ces dernières exigeant, outre un changement de la législation, l'acquisition de nouvelles qualifications techniques.

11 Il convient en outre d'étudier les effets des programmes d'ajustement sur différentes périodes pour déterminer leurs résultats finaux, les effets déflationnistes se produisant plus rapidement.

12 Les données du tableau 1 confirment la baisse du pourcentage des dépenses publiques par rapport au produit national brut (de 63,5\% en 1981/82 à 43,5\% en 1990/91), de même que celle du pourcentage des importations par rapport au produit national brut (de 41,9 \% en 1981/82 à 26,5\% en 1988/89). La part de l'investissement public fixe est de $11,9 \%$ en $1989 / 90$, après avoir été de $22,5 \%$ en $1981 / 82$. Quant au niveau de croissance réelle du produit national brut, il a notablement diminué pour atteindre, en 1988/89, $2,4 \%$. De même, la part de la consommation dans les dépenses publiques a décru, comme on peut le constater. Enfin, la sous-utilisation des ressources antérieure à la réduction des dépenses publiques a décuplé les effets déflationnistes de ces politiques, le taux de chômage apparent atteignant, en 1986, 11,9 \%. La forte croissance du début des années 80 résulte des politiques financières et monétaires expansionnistes, et non de l'augmentation des capacités de production, comme le prouve la baisse constante des niveaux de croissance sectoriels telle qu'elle apparaît dans le tableau 1. 
Tableau 1 : Principaux indices économiques 1981/82-1989/90

\begin{tabular}{|l|r|r|r|r|r|}
\hline \multicolumn{1}{|c|}{ Année } & $81 / 82$ & $85 / 86$ & $88 / 89$ & $89 / 90$ & $90 / 91$ \\
\hline Variables & & & & & \\
\% dépenses publiques par rapport au PNB & 63,5 & 56,4 & 46,6 & 43,7 & 43,5 \\
\% salaires par rapport aux dépenses publiques & & & & & \\
courantes & 24,5 & 25,3 & 28,2 & 27,1 & 23,9 \\
Part des impôts indirects dans le total des & & & & & \\
contributions & 62,4 & 62,4 & 65,6 & 64,1 & 60,9 \\
Taux de croissance du secteur industriel & & 3,5 & $-2,5$ & & \\
Taux de croissance du PNB & 9,9 & 2,6 & 2,4 & 1,3 & \\
Taux de croissance du secteur agricole & & 1,6 & 2,0 & 1,5 & \\
Part des importations dans le PNB & 41,9 & 25,6 & 26,5 & & \\
Part des exportations dans le PNB & 27,0 & 15,7 & 14,9 & & \\
Part de la consommation publique dans le PNB & 17,8 & 16,5 & 13,0 & & \\
Part de la consommation privée dans le PNB & 66,9 & 69,2 & 80,0 & & \\
Part de l'investissement public fixe dans le PNB & 22,5 & 15,0 & 13,1 & 11,9 & \\
Part de l'investissement privé fixe dans le PNB & 7,1 & 7,4 & 6,5 & 6,5 & \\
Taux de croissance de la main-d'œuvre dans le & & & & & \\
secteur & & 9,1 & & &
\end{tabular}

Source : Arab Republic of Egypt, Country economic memorandum, Economic readjustment with growth, 1989, \& IMF, Country rep.1991.

13 De même, les effets des programmes d'ajustement structurel varient d'un pays à l'autre en fonction du volume des ressources humaines disponibles et du poids de la dette extérieure. Ainsi l'Égypte souffre-t-elle d'une forte croissance démographique, d'un taux de dépendance élevé ( $37 \%)$ et de la faiblesse du taux d'activité $(28,4 \% \text { en } 1986)^{3}$, à quoi il faut ajouter le poids de la dette extérieure: la part du service de la dette atteignait, avant son rééchelonnement, $45,5 \%$ en $1989 / 90^{4}$. Autant de facteurs qui entravent la réalisation des effets positifs des programmes d'ajustement, alors qu'apparaissent rapidement leurs effets récessifs et leurs conséquences sociales.

14 Les effets sociaux des programmes d'ajustement sont liés à leur impact sur la croissance : plus cette dernière sera forte, plus l'État sera en mesure de résoudre les problèmes d'emploi et de pauvreté. Il est cependant difficile de cerner les effets positifs de ces programmes. Si des mesures telles que la libération des prix des produits agricoles et industriels peuvent être favorables à la croissance de ces secteurs grâce à l'amélioration de l'efficience économique dans l'allocation des ressources, il reste que, du fait de la sous-utilisation des capacités de production de l'industrie égyptienne et de la faible flexibilité de la structure productive dans ce secteur, ces résultats ne seront pas immédiats.

15 Le tableau 1 fait apparaître les faibles taux de croissance réels dans les principaux secteurs productifs : agriculture et industrie. On sait, d'autre part, que la réduction du déficit de la balance commerciale résulte davantage d'une baisse des importations, que d'une augmentation notable des exportations. Les données du tableau 1 montrent à ce propos que la part des importations dans le produit intérieur brut a diminué de $41,9 \%$ en $1981 / 82$ à $26,5 \%$ en $1988 / 89$, alors que celle des exportations baissait de $27 \%$ à 14,9 \% pendant la même période. D'autre part, si les politiques de relèvement des taux 
d'intérêts créditeurs - jusqu'à 14 et $17 \%$ - ont pu aboutir à l'augmentation de certains types d'épargne au cours des dernières années ${ }^{5}$, la hausse des taux d'intérêt débiteurs jusqu'à $21 \%$ et plus ${ }^{6}$ pourrait avoir des effets récessifs sur l'investissement national, surtout si elle s'accompagnait d'une augmentation des taxes sur la consommation, la part des impôts indirects ayant atteint 60,9 \% de l'ensemble des taxes en 1990/91. Nous avons également noté la baisse de la part de l'investissement public fixe dans le produit national brut (de $22,5 \%$ en $1981 / 82$ à $11,9 \%$ en 1989/90). Il est fort probable, par ailleurs, que la baisse des dépenses d'investissement publiques entraîne une baisse des dépenses d'investissement privées, du fait de l'interdépendance des deux secteurs. Le tableau 1 indique en effet une diminution de la part de l'investissement privé dans le produit national brut (de 7,1 \% en 1981/82 à 6,5\% en 1989/90).

On peut prévoir, au vu de ce qui précède ${ }^{7}$ que les effets récessifs des programmes d'ajustement structurel seront plus automatiques et plus rapides que leurs effets expansionnistes : ces politiques risquent d'avoir des effets négatifs - même pour une période transitoire - sur bon nombre de domaines sociaux en Égypte.

\section{Nature des effets sociaux des programmes des politiques d'ajustement structurel}

Il est difficile de les déterminer au niveau macro-économique. En effet, en dépit du caractère global de la plupart de ces programmes, c'est au niveau individuel que leur impact se fait ressentir. Et alors que leur évaluation s'effectue au moyen d'indicateurs macro-économiques comme le niveau de croissance de la production et de l'investissement ou le déficit budgétaire, ce type d'indicateurs ne permet pas d'en évaluer les effets sociaux: il est nécessaire, pour cela, de procéder à des études sectorielles.

- Les effets sociaux des programmes d'ajustement structurel sont liés à des politiques de redistribution de la richesse entre les individus. Or, il est clair qu'il n'existe pas d'accord théorique sur l'évaluation de ces politiques ${ }^{8}$. En effet, d'après la notion d'équilibre social optimal selon Pareto, une politique donnée est souhaitable si elle permet d'améliorer la situation d'un individu sans affecter le bien-être d'un autre individu. Pour Kaldor, par contre, une mesure économique est acceptable dès lors que plusieurs individus en bénéficient, même au détriment de plusieurs autres, à partir du moment où les "gagnants »sont en mesure d'indemniser les «perdants »et de faire progresser leur situation. Skitovasky, quant à lui, soutient l'impossibilité, pour les "perdants", de contraindre les "gagnants »à maintenir l'état initial de distribution des richesses. En outre, il est difficile d'appliquer les critères qui précèdent à l'étude des effets sociaux des politiques d'ajustement : comment déterminera-t-on, en effet, qui sont les «perdants »et qui sont les «gagnants »dans l'application de ces politiques? Sans compter la multiplicité des fonctions économiques d'un même individu et l'interaction des effets des politiques d'ajustement, que nous examinerons plus loin.

\section{Problématique générale des effets sociaux des programmes d'ajustement}

19 Etudier les effets sociaux des politiques d'ajustement exige tout d'abord d'examiner la manière dont les individus en seront affectés, puis les mesures adoptées et leur impact sur les différentes fonctions économiques des agents, de façon à analyser leurs répercussions sur un ensemble d'indicateurs de bien-être tenant compte des problèmes 
spécifiques de la société égyptienne (pauvreté, écarts importants entre les conditions de vie...)

Un même individu peut être affecté par les mesures d'ajustement selon les diverses fonctions qu'il occupe : producteur, consommateur de biens ou utilisateur de services publics'. L'impact d'une politique donnée sur un même individu diffère selon chacune de ces fonctions. La libération des prix, par exemple, n'aura pas les mêmes effets sur le producteur, le consommateur de biens échangeables et l'utilisateur de services; il en va de même des conséquences de la limitation de l'intervention publique. Le schéma 1 tente de retracer les interactions multiples et les résultats finaux de la politique d'ajustement sur un certain nombre d'indicateurs sociaux tels que le chômage, les revenus réels, la situation alimentaire et sanitaire, l'enseignement. Nous discuterons ces indicateurs dans le contexte de la pauvreté et des disparités de niveaux de vie propres à la société égyptienne.

\section{Politiques d'ajustement affectant les individus}

\section{Effets des politiques d'ajustement sur les producteurs}

21 L'individu-producteur est affecté par les politiques de limitation des dépenses publiques et de transfert des ressources, les politiques de salaires, d'emploi, celles de libération des prix des produits agricoles, ainsi que celles des taux d'intérêt et d'augmentation des impôts à la consommation.

\section{Politiques d'emploi et de salaires}

Les politiques salariales sont essentielles pour la diminution des dépenses publiques, les politiques d'emploi étant quant à elles fondamentales dans le transfert des ressources. Ces deux politiques étant étroitement liées dans le cas de l'Égypte, nous en étudierons conjointement les effets.

Les effets sociaux des politiques d'ajustement structurel

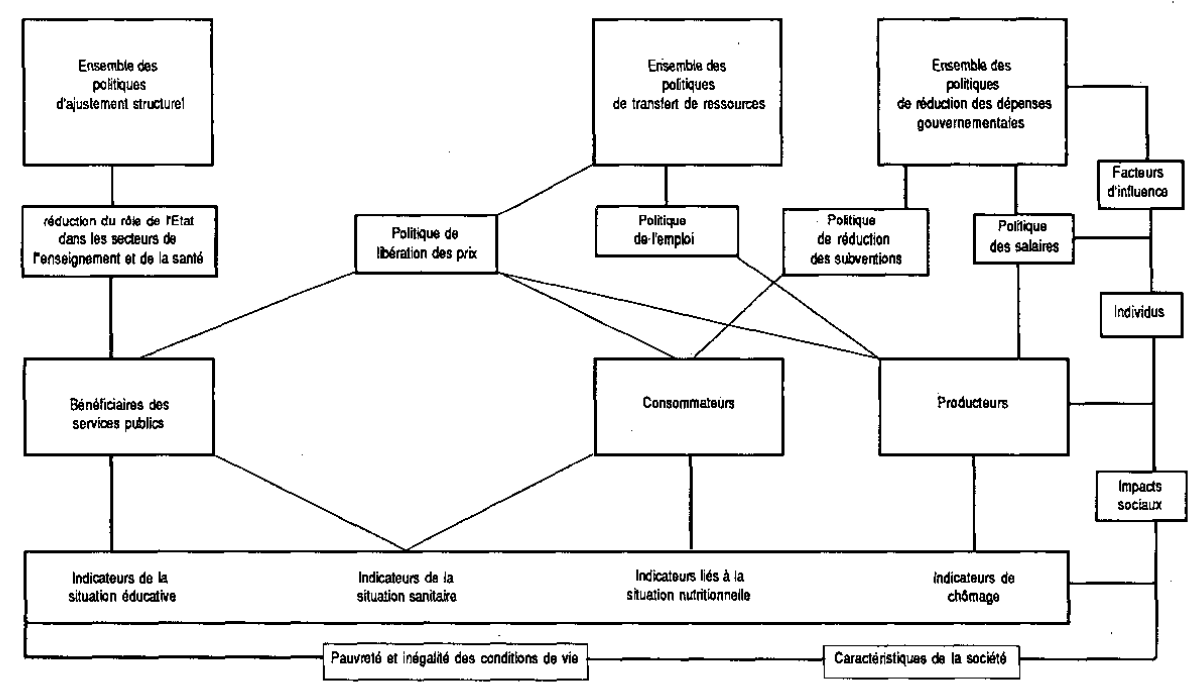


Les salaires occupent en moyenne, dans la décennie 80 , plus du quart des dépenses courantes : $26,1 \%$ (voir tableau 1). La croissance de leur part relative, de $24,5 \%$ à $28,2 \%$ entre 1981/82 et 1988/89, doit être recherchée dans :

1. l'augmentation importante du nombre de fonctionnaires du secteur public : de 2,3 millions en 1981/82 à 3,1 millions en 1986/87;

2. l'augmentation de la masse salariale, qui est passée, de 1981/82 à 1986/87, de 20.781 millions de livres égyptiennes (LE) à 43.687 millions LE, pour atteindre en 1990/91 71.400 millions $\mathrm{LE}^{10}$.

La question de la réduction de la part des salaires dans le budget de l'État est liée à celle de la croissance de l'emploi dans la fonction publique ; cette dernière a contribué à la baisse des salaires réels de $30 \%$ dans la période 1982-86, ainsi qu'à la chute de la croissance des salaires nominaux qui, d'un taux annuel de 2 à $3 \%$ durant la période 89/92, deviendra quasi-nulle entre 92 et 95.

- Les politiques de l'emploi, quant à elles, sont plus complexes : lalégislation en vigueur depuis les années 60 acausé une hypertrophie de l'emploi, dans le secteur public et gouvernemental en particulier où sa croissance aété supérieure à celle de la population (tableau 1). Or, eu égard aux nombreuses restrictions législatives et sociales entravant le licenciement de la main-d'œuvre excédentaire, le gouvernement égyptien a préféré réduire les salaires réels.

De ce qui précède résultent les effets suivants :

27 - une baisse de $10 \%$, durant la période 1973/87, du montant des salaires réels dans le secteur public, alors que ceux du secteur gouvernemental diminuaient de moitié durant la même période (tableau 2).

Tableau 2 : Évolution des salaires réels dans l'économie égyptienne $(1973=100)$

\begin{tabular}{|l|c|r|r|r|r|}
\hline Année & 1973 & 1975 & 1980 & 1985 & 1987 \\
\hline Secteur & & & & & \\
Secteur public & 100 & 83 & 112 & 134 & 102 \\
Secteur privé & 100 & 93 & 10 & 108 & 90 \\
Gouvernemental & 100 & 83 & 80 & 71 & 55 \\
\hline
\end{tabular}

Source : Poverty alleviation and adjustment in Egypt, Document of the World Bank, Report n 8515 EGT, Statistical appendix 21

28 Les salaires réels du secteur privé et ceux du secteur public suivent une évolution opposée. L'indice des salaires dans le secteur public a baissé dans l'agriculture (de 100 en 1973 à 77 en 1987) et dans l'industrie (de 100 en 1973 à 95 en 1987), alors que celui du secteur privé - agriculture et industrie- a atteint respectivement 240 et 135 en 1987. Ces politiques salariales ont eu des répercussions importantes du fait qu'en Égypte, le système de salaires n'est pas lié, d'une manière générale, à la productivité, les primes étant versées à tous les individus au détriment des employés les plus qualifiés. Par exemple, le salaire minimum des employés de sixième échelon a augmenté de $119 \%$ durant la période 1978-84, mais de $19 \%$ seulement pour les employés de premier 
échelon. Il en est résulté la perte d'une part importante de la main-d'œuvre qualifiée du secteur public, qui a préféré émigrer ou aller travailler dans le secteur privé ${ }^{11}$;

- un niveau élevé de chômage parmi les nouveaux diplômés, alternative au licenciement de la main-d'œuvre excédentaire et qui se traduit par :

1. une occupation partielle des postes vacants dans le secteur public et gouvernemental;

2. l'allongement de la période d'attente des nouveaux diplômés avant d'obtenir un poste dans la fonction publique. Ainsi, 1,5 million d'individus ont été embauchés pendant la période du premier plan, soit $20 \%$ des arrivants sur le marché du travail. D'autre part, selon certaines enquêtes auprès des agences pour l'emploi au Caire, la proportion d'inscrits comme demandeurs d'emploi, par rapport aux postes vacants, s'élève à 1 pour $10^{12} \mathrm{du}$ fait de la complexité des formalités d'embauche et de la nécessité de se réinscrire annuellement pour l'obtention d'un poste, durant des périodes pouvant atteindre cinq à six ans. D'où une augmentation du niveau de chômage apparent en Égypte (de 7,4 \% en 1976 à 11,9 \% en 1986). L'on peut faire ici deux remarques: d'une part, puisque le chômage touche surtout les jeunes diplômés, les trois-quarts environ de ceux qui cherchaient un emploi en 1986 avaient entre 16 et 25 ans, et $84 \%$ étaient diplômés du secondaire et au-dessus; d'autre part, le chômage a touché davantage les hommes que les femmes en 1986 par rapport à 1976, diminuant l'inégalité entre les $\operatorname{sexes}^{13}$. Face à ce phénomène, les niveaux de chômage apparent ont ainsi atteint, pour les hommes et les femmes, les chiffres suivants en 1976 et 1986 :

\begin{tabular}{|l|c|c|c|}
\hline \multicolumn{1}{|c|}{ Zones } & Sexe & 1976 & 1986 \\
\hline Urbaines & H & 6,6 & 11,8 \\
& F & 24,5 & 22,7 \\
\hline \multirow{2}{*}{ Rurales } & H & 4,2 & 9,3 \\
& F & 37,3 & 27,3 \\
\hline
\end{tabular}

- la gravité du problème du chômage est liée aux politiques actuelles de limitation de la croissance de la main-d'œuvre dans le secteur public industriel, qui est tombée de 3,6\% en moyenne durant la période 1966/67-1972, à 0,6\% durant la période 1982/83-1984/85. De ce fait, le volume de la main-d'œuvre du secteur public industriel depuis $1982 / 89$ a diminué ${ }^{14}$, de même que le niveau dé croissance de la main-d'œuvre dans le secteur gouvernemental (tableau 1);

31 - nous ne pensons pas que la privatisation résoudra à elle seule les problèmes d'emploi dans l'économie égyptienne, du fait de la faiblesse de la part du secteur privé dans la population active industrielle ( $18,6 \%$ seulement) et de l'élévation de l'intensité capitalistique dans ce secteur. En effet, si le ratio capital/travail n'était que de 211,5 en 1981/82 (aux prix de 70/71) dans le secteur public, il atteignait 545,1 dans les entreprises du secteur privé ${ }^{15}$;

- selon une opinion répandue, la libéralisation des marchés financiers devrait amener une réduction de l'intensité capitalistique dans l'industrie du fait de l'écart entre le prix relatif du capital et celui du travail. Cependant cette libéralisation, même si elle entraînait une baisse de l'intensité capitalistique dans le secteur public industriel, pourrait également produire une hausse de celle du secteur privé industriel grâce à la simplification des formalités d'accès au crédit; en effet, ce secteur devait, avant la réforme, recourir à des ressources propres et aux marchés financiers non officiels ${ }^{16}$. 

fixes comme le coton, le blé, le riz et la canne à sucre (de 1974-80 à 1988). La politique d'ajustement structurel a visé dans ce domaine à supprimer le contrôle gouvernemental sur les prix des produits et des inputs agricoles, sur les superficies consacrées à la production de produits particuliers, sur la vente forcée de certaines denrées à l'État et les restrictions à la commercialisation par le secteur privé des inputs agricoles.

Tableau 3 : Effets des politiques de prix sur la variation du revenu réel des agriculteurs (\%)

\begin{tabular}{|c|c|c|c|c|c|c|c|c|c|c|}
\hline \multirow{2}{*}{ Effets } & \multicolumn{5}{|c|}{ Effets directs (a) } & \multicolumn{5}{c|}{ Effets globaux (b) } \\
\cline { 2 - 10 } & \multicolumn{3}{|c|}{ propriétaires } & locataires & \multicolumn{3}{c|}{ propriétaires } & locataires \\
\hline $\begin{array}{c}\text { superficie } \\
\text { (c) }\end{array}$ & $<1$ & $1-3$ & $3-5$ & $>5$ & & $<1$ & $1-3$ & $3-5$ & $>5$ & \\
\hline $73-79$ & $-25,7$ & 38,3 & $-45,4$ & $-46,5$ & 16,4 & $-50,0$ & $-59,6$ & $-60,9$ & $-53,4$ & 27,9 \\
$80-85$ & $-10,1$ & $-22,7$ & $-29,7$ & $-30,6$ & 13,7 & $-41,0$ & $-50,0$ & $-52,8$ & $-44,3$ & 31,0 \\
\hline
\end{tabular}

Source : Dethier J.J., Trade, exchange rate and agricultural pricing policies in Egypt, Washington D.C., World Bank 1989.

(a) Effets directs : variations du revenu en cas de retrait des subventions en utilisant le taux de change officiel.

(b) Effets globaux : variations du revenu en cas de retrait des subventions en utilisant le taux de change du marché libre des devises.

(c) Superficie des exploitations en feddans : 1 feddan = 0,42 ha

Les valeurs négatives signifient que le revenu des agriculteurs est inférieur en cas de fixation par l'État des prix du blé, du coton, de la canne a sucre et du riz, à ce que serait ce revenu sans fixation étatique des prix. 
Tableau 4 : Effets des subventions alimentaires sur les salaires (transferts de devises) LE/an

\begin{tabular}{|l|r|r|r|r|r|}
\hline $\begin{array}{r}\text { Sources } \\
\text { d'augmentation } \\
\text { du revenu }\end{array}$ & $\begin{array}{r}\text { du } \\
\text { produit }\end{array}$ & $\begin{array}{c}\text { de la } \\
\text { consomm. }\end{array}$ & $\begin{array}{c}\text { du revenu } \\
\text { transtéré }\end{array}$ & $\begin{array}{c}\text { vol. des } \\
\text { dépenses }\end{array}$ & $1 / 3 \%$ \\
\hline Genre & & & & & \\
Zones rurales & 6,13 & 23,8 & 30 & 238,7 & 12,6 \\
Petits producteurs /propriétaires & $-8,93$ & 17,9 & 9,0 & 274,5 & 3,3 \\
Groyens producteurs/propriétaires & $-80,65$ & 7,48 & $-73,1$ & 388,38 & 1,9 \\
$\quad$ propriétaires & 2,38 & 24,17 & 26,56 & 189,87 & 3,9 \\
Main-d'œurre agricole & 1,37 & 21,3 & 22,74 & 303,3 & 7,5 \\
$\quad$ non propriétaire & 3,08 & 20,6 & 23,6 & 317,9 & 7,0 \\
Main-d'œuvre salariée & & & & & \\
Travailleurs à leur compte & & 6,21 & 6,21 & 543,59 & 1,1 \\
Zones urbaines & & 15,58 & 15,58 & 461,46 & 3,4 \\
Travailleurs à leur comple & 15,55 & 15,55 & 513,51 & 2,0 \\
Main-d'œuvre salariée & & & & & \\
Autres & & & & & \\
\hline
\end{tabular}

Source : Alderman H. \& von Braun J., The effects of the Egyptian food ration and subsidy system on income distribution and consumption, International food policy research institute, July 1984, tab. 21.

La principale conséquence de ces mesures est la hausse des prix des produits agricoles. Ainsi, le coton est monté de 115,28 LE la tonne en 1970 à 909,79 LE en 1988; le riz, de 27 à 200 LE la tonne durant la même période ; la tonne de blé a vu son prix croître de 33,72 à $266,7 \mathrm{LE}$ et celui de canne à sucre, de 2,89 à $34 \mathrm{LE}$ pour la période précédemment citée ${ }^{18}$.

Les conséquences sociales les plus importantes de ces politiques touchent au niveau du revenu réel des agriculteurs.

Pour déterminer leur impact, il faut différencier les producteurs agricoles selon la taille de la propriété agricole (cf. tableau 3, qui met en évidence cinq catégories de producteurs). Il semble que les politiques interventionnistes du gouvernement, en particulier pour la fixation des prix des produits agricoles, aient eu des effets négatifs sur le revenu des producteurs, à l'exception des non propriétaires. Leur impact varie selon la taille de la propriété et selon la période: il augmente avec la taille de l'exploitation et le volume de la production; il était plus important durant les années 70 que durant les années 80 , ces dernières ayant connu une hausse des prix de certains produits. On s'attend donc à ce que la libéralisation des prix des produits agricoles accroisse le revenu de toutes les catégories de producteurs, excepté les non propriétaires. À ce propos, il convient de déterminer l'importance des différentes catégories de producteurs de denrées agricoles profitant de la libération des prix. À notre avis, les conséquences de l'ajustement sur les revenus réels varieront selon les groupes, sachant que, en extrapolant les résultats du tableau précédent, les agriculteurs non propriétaires ( $35 \%$ du total des exploitants) ne bénéficieront probablement pas de la libéralisation des prix. Nous pensons donc que pour cette dernière catégorie, l'augmentation du revenu réel sera partiellement annulée par celle des prix des produits alimentaires, comme cela apparaît dans le tableau 4 : le résultat final de l'augmentation des prix alimentaires - en considérant simultanément l'effet sur la consommation et la production - ne sera sans doute positif que pour les gros propriétaires agricoles, c'est-à-dire une part réduite de l'ensemble, la moitié d'entre 
eux $(48,2 \%)$ ne possédant pas plus d'un feddan et le pourcentage des gros propriétaires terriens ne dépassant pas $5,4 \%$ des propriétaires et 3,5 \% du total des producteurs ${ }^{19}$.

Tableau 5 : Variations des prix de quelques produits agricoles subventionnés

\begin{tabular}{|c|c|c|c|}
\hline Produit & Páriode & Prix : plastuel kg & Indice \\
\hline Sucre & $\begin{array}{l}1970-74 \\
1975 \\
1976-80 \\
1980-89\end{array}$ & $\begin{array}{l}15 \\
16 \\
25 \\
30\end{array}$ & $\begin{array}{l}100 \\
107 \\
167 \\
200\end{array}$ \\
\hline Thé & 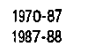 & $\begin{array}{l}137,5 \\
262,5\end{array}$ & $\begin{array}{l}100 \\
191\end{array}$ \\
\hline Riz & $\begin{array}{l}1970-82 \\
1982-86 \\
1986-88 \\
\text { juillet } 1989\end{array}$ & $\begin{array}{r}5 \\
15 \\
30 \\
80\end{array}$ & $\begin{array}{l}100 \\
300 \\
600 \\
700\end{array}$ \\
\hline Farine de blé & $\begin{array}{l}1970-80 \\
1980-85 \\
1985-88\end{array}$ & $\begin{array}{r}5 \\
15 \\
30\end{array}$ & $\begin{array}{l}100 \\
300 \\
600\end{array}$ \\
\hline Fèves & $\begin{array}{l}1970-75 \\
1976-81 \\
19186 \\
1986-88\end{array}$ & $\begin{array}{r}7 \\
10 \\
15 \\
35\end{array}$ & $\begin{array}{l}100 \\
143 \\
214 \\
499\end{array}$ \\
\hline Pain blanc & $\begin{array}{l}1970-80 \\
1980-89\end{array}$ & $\begin{array}{l}0,5 \\
1,0\end{array}$ & $\begin{array}{l}100 \\
200\end{array}$ \\
\hline Michre & 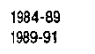 & $\begin{array}{l}2 \\
5\end{array}$ & $\begin{array}{r}400 \\
1000\end{array}$ \\
\hline Lentilles & $\begin{array}{l}1970 \\
1971-80 \\
1981.85 \\
1986-88\end{array}$ & $\begin{array}{r}9 \\
10 \\
12 \\
1120\end{array}$ & $\begin{array}{r}100 \\
111 \\
133 \\
1330\end{array}$ \\
\hline Macaroni & $\begin{array}{l}\text { Jullet } 1982 \\
\text { anvier } 1989 \\
\text { juillet } 1989\end{array}$ & $\begin{array}{r}1150 \\
50 \\
60\end{array}$ & $\begin{array}{r}1567 \\
100 \\
120\end{array}$ \\
\hline Poulet congelé & $\begin{array}{l}\text { janvier } 1989 \\
\text { jullet } 1989\end{array}$ & $\begin{array}{l}320 \\
360\end{array}$ & $\begin{array}{r}100 \\
112,5\end{array}$ \\
\hline EEuts & $\begin{array}{l}\text { janvier } 1989 \\
\text { juilet } 1989\end{array}$ & $\begin{array}{l}14 \\
15\end{array}$ & $\begin{array}{r}100 \\
107,1\end{array}$ \\
\hline Huile (carte verte) & $\begin{array}{l}1970-89 \\
\text { jullet } 1989\end{array}$ & $\begin{array}{l}24,7 \\
31,3\end{array}$ & $\begin{array}{r}100 \\
105,1\end{array}$ \\
\hline
\end{tabular}

Source : calculs effectués à partir d'al-Kholei (1990), cit. tab. 6, et ministère de l'Agriculture.

\section{Effets des politiques d'ajustement structurel sur le consommateur}

\section{Suppression des subventions alimentaires (baisse des dépenses gouvernementales)}

39 Le système des subventions alimentaires en Égypte, destiné à garantir la sécurité alimentaire, est le plus étendu que l'on connaisse dans le monde ${ }^{20}$ : le pourcentage d'individus en possession d'une carte d'approvisionnement atteignait $93 \%$ de la population en 1989 , soit 48,5 millions de personnes. On peut également se procurer sans carte, auprès des coopératives, des produits subventionnés comme les oeufs, l'huile, le sucre, le thé et le poulet surgelé. Un des plus fameux exemples est celui du pain, qui bénéficie d'une double subvention (farine et blé). Le tableau (a) de l'annexe montre l'évolution de la part des subventions alimentaires dans le budget de l'État: elles atteignaient $98 \%$ du total des subventions inscrites au budget en 1973, dont $73 \%$ consacrées à la farine et au blé. Les politiques de réduction des dépenses publiques se sont efforcées de réduire la part des subventions dans le budget de l'État : elles chutent de $31 \%$ en $1980 / 81$ à $5 \%$ seulement en $1988 / 89$, la part des subventions alimentaires dans ce total tombant à $47 \%$ en 1988/89 dont $11 \%$ pour la farine et le blé. 
40 Il en est résulté une nette hausse des prix alimentaires : le prix du pain est passé de 2 piastres (environ 3 centimes) en 1989 à 5 en 91. Quant au kilo de riz, il est passé de 30 piastres en 1988 à 80 en 1989 (tableau 5).

41 Parmi les effets sociaux les plus importants de cette suppression des subventions alimentaires, citons les conséquences sur le niveau nutritionnel des Égyptiens :

- ce système avait contribué à maintenir la consommation calorique par tête à des niveaux élevés - de 2.660 cal en 1969 à 3.501 cal en $1986^{21}$ - proches de ceux en vigueur dans les pays développés, et largement supérieurs à ceux d'autres pays en voie de développement (2150 cal en moyenne) ;

43 - l'augmentation du prix des produits alimentaires de base risque d'avoir des effets négatifs sur la nutrition. En effet (tableau 6), la population urbaine à bas revenus dépense $63 \%$ de son budget dans l'alimentation, dont $25,7 \%$ est subventionnée directement par le gouvernement (carte ou coopérative). $24 \%$ des calories consommées par ces populations proviennent du système de "sécurité alimentaire ", la farine et le pain fournissant $49 \%$ de leur apport calorique quotidien. De même, les ruraux appartenant à la tranche de revenus la plus basse dépensent $68 \%$ de leur budget alimentaire ; le blé et le pain représentent $34 \%$ de leur apport calorique quotidien et le système de sécurité alimentaire contribue pour $16 \%$ à ce dernier et pour $17,9 \%$ du total des dépenses alimentaires. Plus important encore, l'élasticité-prix de certains produits alimentaires, essentiels pour les consommateurs les plus pauvres, est élevée : celle de la farine et du pain «baladi "(populaire) atteint - 2,3 pour les citadins à revenus modestes et $-2,6$ pour les autres habitants des zones, urbaines. L'élasticité-prix de la farine pour les revenus modestes est forte $(-1,9)$, de même que celles du poulet et de la viande qui atteignent respectivement - 2,879 et - 2,158 dans les villes et - 1,58 dans les campagnes $^{22}$. La hausse des prix des produits agricoles a entraîné celle des prix de la ration calorique minimale qui croît de 429 \% et $391 \%$ respectivement pour les ménages des zones urbaines et rurales durant la période 1981/82-89, en utilisant les prix de $81^{23}$; 
Tableau 6 : Données diverses sur l'alimentation (zones rurales et urbaines)

\begin{tabular}{|c|c|c|c|c|c|c|c|c|}
\hline \multirow[t]{2}{*}{ Variables } & \multicolumn{4}{|c|}{$\begin{array}{l}\text { Zones urbaines } \\
\text { classe de revenu }\end{array}$} & \multicolumn{4}{|c|}{$\begin{array}{l}\text { Zones rurales } \\
\text { classe de revenu }\end{array}$} \\
\hline & 1 & 2 & 3 & 4 & 1 & 2 & 3 & 4 \\
\hline $\begin{array}{l}\text { Part des dépenses alimentaires } \\
\text { par rapport aux dépenses } \\
\text { totales }(\%)\end{array}$ & 63 & 56 & 51 & 39 & 68 & 65 & 61 & 48 \\
\hline $\begin{array}{l}\text { Part des dépenses alimentaires } \\
\text { subventionnées }(\%)\end{array}$ & 25,7 & 19,2 & 14,7 & 10,1 & 17,9 & 12,1 & 8,6 & 6,8 \\
\hline Quantité de calories & 2343 & 2761 & 2915 & 3174 & 2357 & 2574 & 2716 & 3149 \\
\hline $\begin{array}{l}\text { Sources de l'apport calorique } \\
\text { - cartes }\end{array}$ & & & & & & & & \\
\hline d'approvisionnement & 19 & 17 & 15 & 12 & 15 & 12 & 10 & 8 \\
\hline - coopératives & 5 & 6 & 6 & 7 & 1 & 1 & 1 & 2 \\
\hline - farine et pain & 49 & 45 & 42 & 35 & 34 & 25 & 19 & 19 \\
\hline - production familiale & - & - & - & - & 8 & 13 & 13 & 14 \\
\hline
\end{tabular}

Source : Données collectées à partir de : Alderman H. \& Von Braun Joachim, The effect of the Egyptian food ration and subsidy system on income distribution and consumption, International food policy Research Institute, Research Report 45, July 1984, table 9 \& table 14.

Tableau 7 : Indices de malnutrition des enfants de 3 à 36 mois

\begin{tabular}{|l|c|c|c|}
\hline \multicolumn{1}{|c|}{ Zones } & Taille / áge & Poids / taille & Poids / âge \\
\hline & & & \\
Urbaines & 25,5 & 1,9 & 8,9 \\
Rurales & 35,1 & 0,9 & 17,0 \\
\hline
\end{tabular}

Source : Sayed H et al., Demography and health survey, 1988. National population council, Cairo, 1989.

44 - la hausse des prix alimentaires entraînera sans aucun doute une ponction importante sur le revenu individuel. Selon les données du tableau 4, les subventions alimentaires représentent un transfert monétaire net en faveur de toutes les couches sociales mentionnées, à l'exception des grands propriétaires de terres agricoles qui ne constituent qu'une part extrêmement faible des agriculteurs ${ }^{24}$. La stabilisation des prix des produits agricoles n'a réduit le revenu réel que pour les moyens et grands propriétaires et les subventions alimentaires ont fait croître celui des autres catégories sociales. Le relèvement des prix du blé et de la farine - qui sont la principale source de calories pour les Égyptiens à revenus modestes - va entraîner une diminution du niveau nutritionnel de ces derniers.

On peut prévoir en second lieu que la réduction des subventions alimentaires va abaisser le niveau nutritionnel national, du fait de l'élasticité-prix élevée des produits à fort contenu calorique par les populations pauvres. Des études sectorielles réalisées au Caire, à Alexandrie et à Assiout, montrent que $25 \%$ des travailleurs de l'échantillon 
étudié avaient cessé d'acheter de la viande, et que $50 \%$ des agriculteurs avaient renoncé à consommer certains légumes à cause de l'augmentation de leur prix ${ }^{25}$;

- la malnutrition, mesurée par les rapports taille/âge, poids/âge, taille/poids (voir tableau 7) pourrait s'aggraver sous l'effet de l'augmentation des prix alimentaires. Il en va de même pour les enfants en âge préscolaire dont $38,4 \%$ souffrent d'anémie (et $11,2 \%$ d'anémie aiguë). $45 \%$ des enfants scolarisés ${ }^{26}, 22,1 \%$ des femmes enceintes et $25,3 \%$ des femmes qui allaitent en souffrent également.

\section{Les politiques de libération des prix (indice des prix)}

Elles figurent au nombre des mesures essentielles d'ajustement structurel et ont pour but de réaliser un transfert des ressources vers les secteurs productifs. Les chiffres suivants montrent l'évolution de l'indice des prix de certains produits et services en 1990 , sur la base des prix de $86 / 87^{27}$ :

$\begin{array}{lrlr}\text { nourriture } & 194,4 & \text { services } & 174,4 \\ \text { vêtements } & 175,4 & \text { santé } & 160,9 \\ \text { loyers et eau } & 121,4 & \text { transports } & 187,8 \\ \text { énergie } & 164 & \text { enseignement } & 194,9 \\ \text { mobilier } & 254,4 & \text { indice du coût de la vie } & 185,8\end{array}$

48 Les études réalisées sur les effets de la réduction des dépenses gouvernementales et la suppression des subventions sur le bien-être concernent trois secteurs : l'électricité, l'énergie et les transports, les services et produits essentiels ${ }^{28}$.

Le prix de l'électricité a augmenté de $30 \%$ en 1990, l'objectif étant de compenser la hausse du coût marginal à l'horizon 1995. Le montant des subventions dans ce secteur atteignait 939,5 millions LE en $87 / 88$, dont $70,4 \%$ allaient aux familles petites consommatrices (moins de $200 \mathrm{kw} / \mathrm{mois}$ ). Notons que $37,2 \%$ de celles-ci consomment moins de $100 \mathrm{kw} /$ mois, tandis que les familles qui en consomment plus de 650 ne bénéficient que de $4,4 \%$ des subventions ${ }^{29}$. On est en droit de s'interroger sur l'effet de l'augmentation du prix de l'électricité sur le niveau de vie des ménages modestes, les plus sensibles à la suppression des subventions; certaines, d'ailleurs, en particulier dans les zones rurales, n'en pâtiront pas puisqu'elles ne consomment pas d'électricité : c'est le cas de 3,9\% des citadins et de $21,1 \%$ des ruraux ${ }^{30}$.

50 Si le quart environ des ruraux ne risque pas d'être touché par la hausse du prix de l'électricité, celle du kérosène, en revanche, aura pour eux d'importantes conséquences. Le kérosène en effet, dérivé du pétrole, représente $13 \%^{31}$ des sources d'éclairage en milieu rural. Dans les familles modestes, il sert également de combustible pour la cuisson des aliments. Avec une élasticité-revenu négative, il est surtout utilisé par les ménages les plus pauvres.

51 En ce qui concerne les transports entre agglomérations, on peut s'attendre à ce que leur prix augmente au détriment des faibles revenus, les catégories aisées habitant les villes proches de la capitale et utilisant leur voiture privée. Cela reste vrai en ce qui concerne la suppression des subventions aux transports dans l'agglomération même ${ }^{32}$ : les personnes jouissant de hauts revenus utilisent leur voiture pour leurs déplacements 
privés, celles à revenus modestes Ont recours aux transports publics. La hausse du prix des transports publics se répercutera donc sur la consommation de ces dernières, qui essaieront de réduire leurs déplacements et les limiteront aux déplacements professionnels.

Nous n'aborderons pas les effets de l'augmentation du prix de l'eau, en dépit de l'évolution qui a eu lieu depuis 1985 (mise en place d'un système visant à tenir compte de la quantité consommée), une part importante des familles rurales ne bénéficiant pas encore de l'eau courante dans leur habitation ( $44,1 \%$ en milieu rural, $7,7 \%$ en milieu urbain) $)^{33}$.

\section{Effets des politiques d'ajustement sur les usagers des services publics}

\section{Politiques de limitation du rôle de l'État en matière de santé et d'enseignement}

\section{Santé}

Après l'instauration d'un service public de soins gratuits, symbole de la politique sanitaire des années 60 qui s'est perpétuée dans les années 70, un net changement est intervenu vers le milieu des années 80 : à partir de cette époque, on a commencé à encourager le secteur privé et à appliquer un système de compensation des dépenses du secteur public sur la base des dépenses réelles. De même, les prix des médicaments produits localement ont été relevés de façon à rétablir la rentabilité des entreprises ${ }^{34}$. La santé est un des secteurs les plus touchés par la réduction des dépenses publiques : leur part est passée de $5 \%$ en 1965 à $1,9 \%$ en 1989/90. Quant aux dépenses de santé par tête, elles sont passées de 6,6 LE en 83/84 à 4,38 LE en 1987/88 et en prix constants, en plus des niveaux de croissance des dépenses de santé en prix constants depuis $1985 / 86^{35}$.

54 Le tableau (b) de l'annexe reflète la diminution de la part des dépenses de santé par rapport au produit national brut : cette part n'est que de $0,6 \%$ en $89 / 90$, à un moment où l'OMS (Organisation mondiale de la Santé) estime à $5 \%$ la part devant être consacrée à ce secteur pour garantir « la santé pour tous ». Diminution constatée également dans le pourcentage des dépenses publiques de santé, qui n'était plus que de $1,9 \%$ en 1989/90 après avoir été de $4,7 \%$ en 1966/67.

Les politiques de compression des dépenses publiques de santé se font sentir surtout sur l'efficacité de ce secteur. La part des salaires atteint $69,3 \%$ du total des dépenses de santé en 1988/89. Etant donné la difficulté de réduire la masse salariale, ce sont les dépenses qui sont réduites. En effet, comme le montre le tableau (c) de l'annexe, le pourcentage par tête des dépenses en équipements médicaux a diminué de $54 \%$ durant les années 80 , avec pour conséquence une baisse du nombre de lits par habitant (de 1,66 en 1967 à 1,1 en 1989/90) ${ }^{36}$ et une dégradation sensible du niveau des services sanitaires publics (pénuries de médicaments et d'instruments médicaux).

La réduction de l'intervention de l'État dans ce secteur dynamique a provoqué une baisse de la demande de services sanitaires qui, selon de nombreuses études, est élastique pour les couches à revenus modestes. Par conséquent, l'imposition de taxes sur les services sanitaires publics, concomitante de la libération des prix, en particulier 
des produits alimentaires, ne peut qu'entraîner une baisse du revenu réel par tête ; les Égyptiens à revenus modestes préféreront alors satisfaire leurs besoins de consommation plutôt qu'améliorer leur niveau de vie sanitaire, surtout si les prix des produits alimentaires de base sont à la hausse. De plus, la demande de services de santé implique un investissement humain, dont toute augmentation des prix des inputs sanitaires ou des produits alimentaires relèvera le coût marginal. Et avec la baisse du revenu réel, l'utilité marginale des dépenses de santé diminuera, conduisant à une réduction de la demande des services de santée ${ }^{37}$.

Tableau 8 : Niveau de mortalité infantile et maternelle (1990)

\begin{tabular}{|l|c|c|}
\hline Pays & $\begin{array}{c}\text { Niveau de mortalité } \\
\text { maternelle (pour 1.000 } \\
\text { naissances) }\end{array}$ & $\begin{array}{c}\text { Niveau de mortalité } \\
\text { infantile }\end{array}$ \\
\hline Egypte & 300 & 61 \\
Irak & 250 & 63 \\
Jordanie & 200 & 40 \\
Tunisie & 200 & 48 \\
Syrie & 200 & 44 \\
Algérie & 210 & 68 \\
Panama & 60 & 22 \\
Koweit & 30 & 17 \\
Qatar & 140 & 29 \\
Bahrein & 80 & 14 \\
Argentine & 140 & 31 \\
Mexique & 150 & 40 \\
lle Maurice & 130 & 22 \\
Cuba & 54 & 11 \\
\hline
\end{tabular}

57 L'importante diminution de la demande de services sanitaires chez les individus à revenus modestes s'explique de deux façons :

1. d'une part, la plupart des maladies graves dont souffrent les Égyptiens (bilharziose, par exemple, ou maladies infectieuses comme la typhoïde, la paratyphoïde, l'hépatite) sont liées à la pauvreté, aux mauvaises conditions de vie, à la malnutrition des enfants et des femmes. La réduction du revenu réel des classes défavorisées - celles qui sont le plus exposées à ces maladies - et l'augmentation du prix des services sanitaires sont susceptibles de faire obstacle à l'éradication de ces maladies ;

2. d'autre part on constate, comparativement à d'autres pays du Moyen-Orient et à certains pays africains, la position défavorable des indicateurs de santé en Égypte (tableau 8) ${ }^{38}$.

\section{Enseignement}

Ce secteur a également été l'objet de mesures d'ajustement structurel pour limiter les conséquences du faible taux de croissance des dépenses publiques. Le tableau (b) de l'annexe montre la baisse de la part des dépenses publiques affectées à ce secteur : elles passent de $15,3 \%$ en 1966/67 à $10 \%$ en 1989/90. De même, par rapport au produit 
national brut, ces dépenses sont passées de 4,4\% en 1966/67 à 3,3\% en 1989/90. La réduction de l'intervention de l'État dans ce secteur se traduit par $^{39}$ : d'ouvriers, $6,7 \%$ chez les fils d'employés et enfin $3,4 \%$ chez les enfants de commerçants. Pour ces catégories de population, l'enfant qui quitte le système scolaire devient une source de revenus pour sa famille. Ce problème ne se rencontre pas dans les milieux aisés. Au total, seuls 65,6\% des garçons et 59,2\% des filles parviennent en sixième. Quant à ceux qui obtiennent le diplôme de fin d'études primaires, leur pourcentage ne dépasse pas $74,9 \%$ pour les garçons et $41 \%$ pour les filles :

63 L'augmentation des coûts et la réduction des moyens matériels dans l'enseignement a également entraîné des disparités criantes de niveaux depuis l'instauration d'un système de rotation par groupes. Ainsi, dans les écoles à groupe unique, le cours dure 45 minutes et la journée scolaire 5 heures et demie ; dans les établissements où deux groupes se succèdent, le cours dure 40 minutes et la journée scolaire 4 heures et demie ; les écoles où alternent trois groupes limitent les cours à 35 minutes et des journées scolaires à 2 heures cinquante. Ce système étant appliqué aux écoles des quartiers populaires à forte densité de population, il ne peut que conduire à d'importantes disparités de niveau entre les enfants de familles aisées et ceux des milieux modestes ${ }^{41}$. 


\section{Impact social des politiques d'ajustement structurel à la lumière de quelques problèmes sociaux particuliers}

La gravité des problèmes ci-dessus évoqués est susceptible d'augmenter du fait de leur coexistence avec d'autres problèmes sociaux :

1. a) l'augmentation du degré de pauvreté dans la société égyptienne ;

2. b) l'impact des programmes d'ajustement structurel sur les couches pauvres en Égypte ;

3. c) Les disparités évidentes dans les indicateurs de qualité de la vie en Égypte.

\section{Un seul élevé de la pauvreté}

65 Le tableau (d) de l'annexe, qui synthétise les résultats de différentes études sur le pourcentage de familles pauvres en Égypte, fait apparaître les faits suivants :

1) Malgré les efforts de redistribution des revenus à travers les politiques sociales réalisées dans le domaine de la santé, de l'éducation et de l'emploi en Égypte, et malgré des niveaux de croissance très élevés pour l'économie égyptienne durant la période 1974/81 (9 \% en moyenne), malgré enfin l'augmentation de l'émigration vers les pays du Golfe, on compte encore en Égypte une proportion non négligeable de familles pauvres.

67 2) Selon les estimations les plus basses du tableau (d), il apparaît que le pourcentage de familles pauvres a augmenté dans les villes : en 1984, on en compte $34 \%$ alors qu'on en comptait $30 \%$ en $1958 / 59^{42}$. Nous constatons en revanche une légère baisse de ce pourcentage dans les campagnes durant la même période ( $35 \%$ en 1958/59 et 33,7 \% en 1984). Une baisse sensible de ces chiffres apparaît encore en $1981 / 82$ : ils passent à $24,2 \%$ et $22,5 \%$ dans les campagnes et les villes respectivement, selon les estimations les plus basses, et à $29,7 \%$ et $30,4 \%$ selon les plus élevées. Les migrations professionnelles vers les pays du Golfe sont à notre avis à l'origine de cette tendance. En utilisant les prix du marché des produits alimentaires, le pourcentage de familles pauvres peut atteindre $47,2 \%$ dans les campagnes et $51,1 \%$ dans les villes.

Si ces données sont exactes, cela signifie que les programmes d'ajustement structurel vont être appliqués dans une société dont la population est constituée pour moitié de citoyens vivant en dessous du seuil de pauvreté.

\section{Effets des programmes d'ajustement structurel sur les classes pauvres}

Une étude réalisée par Karima Korayem sur les caractéristiques économiques et sociales des classes pauvres en Égypte fait apparaître les résultats présentés dans le tableau $9^{43}$. Il n'est pas dans notre propos de discuter ici ces données. Nous formerons seulement l'hypothèse qu'il s'agit là des classes les plus défavorisées. Ces catégories seront-elles touchées par les programmes d'ajustement structurel?

70 1) Le nombre des chômeurs comptabilisés parmi les pauvres risque d'augmenter avec les politiques de transfert de ressources et, en particulier, les politiques d'emploi accroissant le nombre de pauvres en Égypte. 
71 2) Les pensionnés et bénéficiaires d'assurances sociales peuvent voir leur niveau de vie se dégrader sous l'impact de la libération des prix, de la limitation des subventions alimentaires et de l'intervention de l'État dans certains secteurs - la santé notamment ${ }^{44}$, du fait :

1. de la baisse des salaires réels dans les secteurs public et gouvernemental, réduisant le pouvoir d'achat des titulaires de pensions ;

2. de la baisse des ressources de l'État et de la tendance de ce dernier à emprunter sur les fonds des assurances sociales (au taux de $30 \%$ en 87$)^{45}$;

3. de la baisse de la capacité financière des services sociaux, cela en raison de deux facteurs : d'une part, la compression des dépenses gouvernementales et la réduction de la part des dépenses consacrées aux services sociaux (de 1,4\% en 1980 à 1,1\% en 1988); d'autre part, l'augmentation de l'espérance de vie en Égypte (de 46,1 ans en 1960 à 60,3 ans en 1990) ${ }^{46}$. Il résulte de ces deux facteurs une baisse de la valeur des équipements par tête (de 1,74 LE en 1980/81 à 0,33 LE en 1989/90), c'est-à-dire une baisse de $81 \%$. La stagnation à 8,5 LE du montant des retraites depuis 1981 est un facteur supplémentaire de dégradation du pouvoir d'achat des individus concernés, d'autant que les retraités les plus défavorisés ne touchent guère plus de 3 ou 6 LE (cf. loi no 30 de 1977 sur la Sécurité sociale $)^{47}$;

4. de la réduction de main-d'œuvre dans le secteur public, qui entraîne une augmentation des charges financière pesant sur ce secteur du fait de la hausse des indemnités de chômage.

Tableau 9 : Part des personnes défavorisées dans différentes catégories de population

\begin{tabular}{|c|c|c|}
\hline Catégories & Nombre & $\begin{array}{c}\text { Part des } \\
\text { défavorisés dans } \\
\text { chaque catégorie }\end{array}$ \\
\hline Chômeurs & 1.429 .956 & 100 \\
\hline Bénéficiaires de retraites & 143.926 & 100 \\
\hline Assurés sociaux & 20.217 & 100 \\
\hline $\begin{array}{l}\text { Travailleurs du secteur public } \\
\text { (second échelon et moins) }\end{array}$ & 4.235 .762 & 90 \\
\hline $\begin{array}{l}\text { Main-d'œuvre agricole non-propriétaire } \\
\text { Producteurs agricoles }\end{array}$ & 1.657 .805 & 100 \\
\hline $\begin{array}{l}\text { propriétaires de } 3 \text { feddans et moins } \\
\text { Travailleurs du secteur privé }\end{array}$ & 2.623 .000 & 100 \\
\hline non officiel & 876.068 & 70 \\
\hline Travailleurs du secteur privé officiel & 845.810 & 40 \\
\hline Total & 11.832 .544 & 100 \\
\hline
\end{tabular}

72 3) Pour les travailleurs du premier ou deuxième échelon du secteur public, les programmes d'ajustement structurel risquent d'avoir les conséquences suivantes :

1. baisse de leur salaire réel (à supposer qu'ils n'aient pas de second emploi) ;

2. baisse du revenu réel suite à la suppression des subventions alimentaires (la main-d'œuvre salariée est la première catégorie bénéficiant de ces subventions en milieu urbain);

3. coût accru des services éducatifs et de santé ; 
4. crainte de la perte de l'emploi au cas où les politiques prévues en la matière seraient intégralement appliquées.

4) Pour les travailleurs agricoles ne possédant pas de terre, une détérioration des conditions de vie est probable du fait de la suppression des subventions alimentaires, les politiques de fixation des prix alimentaires entraînant une augmentation de 13,5\% de leur revenu réel. En revanche, cette catégorie ne devrait pas être trop touchée par la limitation du rôle de l'État dans le secteur de l'enseignement, du moins pour les plus démunis, chez lesquels l'analphabétisme ou la non scolarisation sont répandus. Pour ce qui est du secteur de la santé, cette catégorie a recourt essentiellement aux services sanitaires publics, et toute déficience dans ce domaine ne peut que se répercuter sur elle. L'augmentation générale du coût de la vie - exception faite des dépenses alimentaires - aura quant à elle peu d'impact, les exigences en termes d'équipements étant limitées chez cette population du fait de son extrême pauvreté.

5) Pour les producteurs agricoles propriétaires de 3 feddans ou moins, les programmes d'ajustement structurel sont susceptibles d'avoir des répercussions négatives sur leurs conditions de vie du fait de :

1. la réduction des subventions alimentaires, qui représentaient pour ces catégories un transfert monétaire net (tableau 4);

2. la limitation du rôle de l'État dans les domaines de la santé et de renseignement, en particulier depuis l'extension aux zones rurales du système de compensation des dépenses de santé, dont une des composantes vise à encourager le secteur privé ${ }^{48}$. De plus, la conscience de l'importance de l'enseignement est croissante dans cette catégorie de population (notamment si on la compare à la catégorie précédente).

6) Les travailleurs à revenus modestes du secteur privé officiel risquent, eux, d'être touchés de plein fouet par la limitation du rôle de l'État dans les secteurs de l'enseignement et de la santé, la réduction des subventions alimentaires et les politiques d'augmentation du coût de la vie. En effet, leurs conditions de travail exigent une apparence sociale déterminée qui leur fera supporter des charges matérielles croissantes, la nature du travail dans le secteur privé ne leur permettant pas d'exercer un second métier. 
Tableau 10 : Indicateurs de qualité de vie (zone urbaine, zone rurale)

\begin{tabular}{|c|c|c|c|c|c|c|c|c|}
\hline \multicolumn{2}{|c|}{ Gouvernorat } & \multicolumn{2}{|c|}{$\begin{array}{c}\text { Taux de } \\
\text { morralité } \\
\text { infantile } \\
\text { Classement (\%) }\end{array}$} & \multicolumn{2}{|c|}{$\begin{array}{c}\text { Taux } \\
\text { d'analphabétisme }\end{array}$} & \multicolumn{2}{|c|}{$\begin{array}{c}\text { Logements } \\
\text { approvisionnés en eau } \\
\text { potable } \\
\text { Classement (\%) } \\
\end{array}$} & $\begin{array}{c}\text { Indicateurs } \\
\text { synthéliques } \\
\text { de qualité } \\
\text { de la vie }\end{array}$ \\
\hline Le Caire & & 74 & 31 & 31 & 100 & 95 & 96 & 73 \\
\hline Alexandrie & & 61 & 33 & 33 & 88,5 & 99 & 95 & 79,5 \\
\hline Port Saïd & & 49 & 32 & 32 & 94 & 100 & 100 & 87 \\
\hline Suez & & 14 & 34 & 34 & 81 & 99 & 95 & 92 \\
\hline Damielte & u & 39 & 76,2 & 36 & 69 & 99 & 95 & 80 \\
\hline & R & 67 & 57 & 47 & 55 & 95 & 100 & 71 \\
\hline Daqahliya & u & 47 & 68,6 & 36 & 69 & 98 & 90 & 76 \\
\hline & $\mathrm{R}$ & 51 & 84 & 53,5 & 41 & 75 & 73 & 66 \\
\hline Charqiya & u & 57 & 59 & 35 & 75 & 95 & 76 & 70 \\
\hline & $\mathbf{R}$ & 67 & 57 & 56 & 36 & 59 & 51 & 48 \\
\hline Qaliubiya & $\tilde{u}$ & 91 & 26,7 & 38 & 56 & 89 & 48 & 43,5 \\
\hline & $\mathbf{R}$ & 80 & 34 & 52 & 79 & 40 & 25 & 46 \\
\hline Kaft al- & u & 53 & 62,8 & 45 & 12,5 & 97 & 86 & 54 \\
\hline Chaykn & $\mathrm{R}$ & 42 & 100 & 65 & 17 & 82 & 82 & 66 \\
\hline Gharbiya & u & 67 & 49,5 & 33 & 88,5 & 95 & 76 & 71 \\
\hline & $\mathrm{R}$ & 75 & 43 & 55 & 38 & 66 & 60 & 47 \\
\hline Menufiya & u & 73 & 43,8 & 37 & 62,5 & 84 & 24 & 43 \\
\hline & $\mathbf{R}$ & 92 & 14 & 51 & 47 & 57,5 & 49 & 37 \\
\hline Buhayra & u & 90 & 24 & 41 & 37,5 & 91 & 57 & 39,5 \\
\hline & $\mathrm{R}$ & 57 & 74 & 62 & 23 & 64 & 57,5 & 51,5 \\
\hline Isma'liya & u & 52 & 63,8 & 32 & 94 & 92 & 62 & 73 \\
\hline & $\mathbf{R}$ & 52 & 83 & 26 & 100 & 48 & 36 & 73 \\
\hline Giza & U & 85 & 32,4 & 34 & 81 & 81 & 10 & 41 \\
\hline & $\mathrm{R}$ & 79 & 36 & 59 & 30 & 50 & 38 & 35 \\
\hline Beni Suef & $\dot{u}$ & 90 & 27,6 & 45 & 12,5 & 87 & 38 & 26 \\
\hline & R & 78 & 38 & 70 & 6 & 62 & 55 & 33 \\
\hline Fayoum & $U$ & 84 & 33,3 & 47 & 1 & 99 & 95 & 43 \\
\hline & R & 74 & 45 & 73 & $i$ & 86 & 88 & 45 \\
\hline Miniya & U & 85 & 32,4 & 39 & 50 & 86 & 33 & 38 \\
\hline & $\mathbf{R}$ & 71 & 50 & 72 & 2 & 36 & 19 & 24 \\
\hline Assiut & U & 89 & 28,6 & 39 & $12, \overline{5}$ & 83 & 19 & 32,5 \\
\hline & $\mathrm{R}$ & 82 & 31 & 70 & 6 & 45 & 31,5 & 23 \\
\hline Suhag & $u$ & 89 & 28,6 & 45 & 19,5 & 82 & 14 & 18 \\
\hline & $\mathrm{A}$ & 61 & 67 & 71 & 4 & 29 & 9,5 & 27 \\
\hline Qena & u & 105 & 13,3 & 44 & 19 & 79 & 1 & 11 \\
\hline & $\mathrm{R}$ & 62 & 65,5 & 69 & 8,5 & 22 & 1 & 25 \\
\hline Aswan & us & 119 & 1,1 & 37 & 62,5 & 94 & 71 & 45 \\
\hline & $\mathrm{R}$ & 100 & $i$ & 52 & 79 & 78 & 77 & 52 \\
\hline
\end{tabular}

$\mathrm{U}=$ Urbain $\mathrm{R}=$ Rural. Source : CAPMAS.

76 7) Enfin, les travailleurs démunis du secteur privé non officiel peuvent essentiellement pâtir de la réduction des subventions alimentaires. Et si l'éducation ne constitue pas pour eux un poste de dépense significatif, ils ne sont pas en mesure, en revanche, d'assumer la privatisation des services de santé ni une augmentation des prix des autres services publics.

\section{Disparités dans les conditions de vie}

Elles dépendent de deux éléments : d'une part, le lieu de résidence (zones rurales ou zones urbaines), d'autre part, le niveau socio-économique. Les indicateurs des programmes d'ajustement structurel s'appuyant sur des moyennes et ne reflétant pas les inégalités sectorielles et géographiques, ces programmes risquent de les aggraver. La politique d'affectation des ressources en Égypte est liée à un type de développement connu sous le nom de " développement par le haut $"^{49}:$ il s'agit de concentrer les efforts de développement dans les grands centres urbains ou les capitales régionales, à partir desquelles ils doivent se diffuser. Ce modèle de développement a prouvé son échec en Égypte, si l'on se réfère à la baisse du volume global des investissements et à leur concentration au Caire et à Alexandrie, ainsi qu'au sous-développement des autres régions.

78 Nous avons tenté, dans les tableaux 10 et 11, de mettre en évidence ce phénomène en utilisant trois indicateurs : le niveau d'analphabétisme, le niveau de mortalité infantile et le pourcentage d'habitations reliées à un réseau d'eau potable. Les données de ces tableaux mettent en évidence des disparités manifestes entre les gouvernorats urbains 
(79), ceux de Basse $(51,6)$ et de Haute-Égypte $(30,1)$, ainsi qu'entre zones urbaines $(58,5)$ et rurales $(44,5)$.

79 Nos craintes quant à une augmentation de l'inégalité des conditions de vie reposent sur le fait que les politiques d'ajustement structurel affectent les différentes catégories sociales à la ville comme à la campagne, alors que les solutions proposées pour atténuer l'impact de ces politiques se limitent aux grands centres urbains au Caire. Les programmes du Fonds Social, par exemple, ont surtout pour objectif de limiter le chômage dans le secteur public, c'est-à-dire dans des unités concentrées en zone urbaine, en négligeant les autres secteurs.

Tableau 11 : Indicateurs de qualité de vie

\begin{tabular}{|c|c|c|c|c|c|c|c|}
\hline Gouvernorat & \multicolumn{2}{|c|}{$\begin{array}{c}\begin{array}{c}\text { Niveau de } \\
\text { mortalité } \\
\text { infantile }\end{array} \\
\text { Classement (\%) }\end{array}$} & \multicolumn{2}{|c|}{$\begin{array}{c}\text { Pourcentage } \\
\text { d'analphabétisme } \\
\text { Classement (\%) }\end{array}$} & \multicolumn{2}{|c|}{$\begin{array}{l}\text { Logements } \\
\text { approvisionnés } \\
\text { en eau potable } \\
\text { Classement }(\%)\end{array}$} & $\begin{array}{l}\text { Indicateurs de } \\
\text { qualité } \\
\text { de vie }\end{array}$ \\
\hline $\begin{array}{l}\text { Le Caire } \\
\text { Alexandrie } \\
\text { Port Saïd } \\
\text { Suez } \\
\text { Damiette } \\
\text { Daqahliya } \\
\text { Charqiya } \\
\text { Qaliubiya } \\
\text { Kafr al-Chaykh } \\
\text { Gharbiya } \\
\text { Menufiya } \\
\text { Buhayra } \\
\text { Isma'iliya } \\
\text { Giza } \\
\text { Beni Suef } \\
\text { Fayoum } \\
\text { Miniya } \\
\text { Assiut } \\
\text { Suhag } \\
\text { Dena } \\
\text { Aswan }\end{array}$ & $\begin{array}{r}74 \\
61 \\
49 \\
14 \\
59 \\
50 \\
65 \\
84 \\
45 \\
72 \\
89 \\
64 \\
52 \\
82 \\
81 \\
77 \\
74 \\
84 \\
67 \\
27 \\
107\end{array}$ & $\begin{array}{r}35 \\
49 \\
38 \\
100 \\
52 \\
61 \\
45 \\
25 \\
67 \\
59 \\
45 \\
46 \\
59 \\
27 \\
28 \\
32 \\
35 \\
25 \\
43 \\
38 \\
1\end{array}$ & $\begin{array}{l}31 \\
33 \\
32 \\
34 \\
44 \\
49 \\
51 \\
46 \\
60 \\
47 \\
48 \\
57 \\
26 \\
44 \\
63 \\
67 \\
65 \\
62 \\
65 \\
63 \\
46\end{array}$ & $\begin{array}{r}88 \\
83 \\
85 \\
80 \\
56 \\
44 \\
39 \\
51 \\
17 \\
49 \\
46 \\
24 \\
100 \\
56 \\
10 \\
1 \\
5 \\
12 \\
5 \\
10 \\
51\end{array}$ & $\begin{array}{r}95 \\
99 \\
100 \\
99 \\
96 \\
81 \\
68 \\
62,5 \\
86 \\
77 \\
63 \\
71 \\
71 \\
69 \\
68 \\
89 \\
47 \\
56 \\
42 \\
35 \\
85\end{array}$ & $\begin{array}{r}92 \\
98,5 \\
100 \\
98,5 \\
94 \\
71 \\
51 \\
42 \\
78 \\
65 \\
43 \\
55 \\
55 \\
52 \\
51 \\
83 \\
34 \\
32 \\
11 \\
1 \\
77\end{array}$ & $\begin{array}{l}72 \\
77 \\
74 \\
93 \\
67 \\
59 \\
45 \\
39 \\
54 \\
51 \\
36 \\
42 \\
71 \\
45 \\
30 \\
39 \\
25 \\
23 \\
20 \\
16 \\
43\end{array}$ \\
\hline
\end{tabular}

Source : CAPMAS Statistiques de naissances et de décès. 
Tableau a : Évolution de divers indicateurs sur les subventions à la consommation 1973-1988/1989 (\%)

\begin{tabular}{|l|c|c|c|c|}
\hline \multicolumn{1}{|c|}{ Année } & 1973 & $1980 / 81$ & $1984 / 85$ & $1988 / 89$ \\
\hline Subventions & 9 & 31 & 10 & 5 \\
$\begin{array}{l}\text { Part des subventions dans les } \\
\text { dépenses publiques }\end{array}$ & 98 & 66 & 56 & 47 \\
$\begin{array}{l}\text { Part des subventions } \\
\text { alimentaires dans les } \\
\text { subventions totales }\end{array}$ & 73 & 35 & 31 & 11 \\
$\begin{array}{l}\text { Part des subventions à la } \\
\text { farine el au blé dans les } \\
\text { subventions totales }\end{array}$ & 74 & 53 & 55 & 23 \\
\hline $\begin{array}{l}\text { Part des subventions à la } \\
\text { farine et au blé dans les } \\
\text { subventions alimentaires }\end{array}$ & & & & \\
\hline
\end{tabular}

Source : al-Kholei, «Objectives and implications of Egyptian food policies », Égypte Contemporaine, Société égyptienne d'économie politique, avril 1990.

Tableau b : Part des dépenses de services sociaux dans le produit national brut et les dépenses publiques $(\%)$

\begin{tabular}{|l|c|c|}
\hline \multicolumn{1}{|c|}{ Année } & $1966 / 1967$ & $1989 / 1990$ \\
\hline Dépenses & & \\
\hline $\begin{array}{l}\text { Dépenses de santé } \\
\text { PNB } \\
\text { Dépenses publiques }\end{array}$ & 1,3 & 0,6 \\
Dépenses éducatives & 4,7 & 1,9 \\
PNB \\
Dépenses publiques & 4,4 & 3,3 \\
\end{tabular}

Source : estimations tirées du Livre d'or du ministère de la Santé 1936-1986 et données des comptes de clôtures du ministère des Finances. 
Tableau c : Part des dépenses d'équipements par habitant dans les secteurs de la santé, des services sociaux et de l'enseignement 1980/81-1989/90 (en LE)

\begin{tabular}{|c|c|c|c|}
\hline Secteur & $1980 / 1981$ & $1985 / 1986$ & $1989 / 1990$ \\
\hline $\begin{array}{l}\text { Santé } \\
\text { Indice }\end{array}$ & $\begin{array}{r}1,26 \\
100\end{array}$ & $\begin{array}{r}0,95 \\
99\end{array}$ & $\begin{array}{r}0,55 \\
46\end{array}$ \\
\hline $\begin{array}{l}\text { Services sociaux } \\
\text { Indice }\end{array}$ & $\begin{array}{l}1,74 \\
100\end{array}$ & $\begin{array}{l}0,61 \\
35,1\end{array}$ & $\begin{array}{r}0,33 \\
19\end{array}$ \\
\hline $\begin{array}{l}\text { Enseignement } \\
\text { Indice }\end{array}$ & $\begin{array}{r}7,14 \\
100\end{array}$ & $\begin{array}{l}4,73 \\
66,2\end{array}$ & $\begin{array}{r}2,37 \\
33\end{array}$ \\
\hline $\begin{array}{l}\text { Enseignement supérieur } \\
\text { Indice }\end{array}$ & $\begin{array}{r}146,67 \\
100\end{array}$ & $\begin{array}{r}158,86 \\
108,3\end{array}$ & $\begin{array}{r}123,9 \\
81,7\end{array}$ \\
\hline
\end{tabular}

Source : World Bank, Property alleviation and adjustment in Egypt, 1990.

Tableau d: Pourcentage de familles pauvres en Égypte.

\begin{tabular}{|l|c|c|}
\hline \multicolumn{1}{|c|}{ Année } & $\begin{array}{c}\text { Pourcentage de } \\
\text { familles pauvres (\%) }\end{array}$ & $\begin{array}{c}\text { Nb de familles pauvres } \\
\text { (milliers) }\end{array}$ \\
\hline $1958 / 1959$ & 35 & 1161 \\
Campagne & 30 & 597 \\
Villes & & 1833 \\
$1974 / 1975$ & 44 & 1076 \\
Campagne & 34,5 & $1023-1240$ \\
Villes & & $756-1196$ \\
$1981 / 1982$ & $24,2-29,7$ & \\
Campagne & $22,5-30,4$ & $1476,1-2067,4$ \\
Villes & & $1444,7-2171,2$ \\
$1983 / 1984$ & $33,7-47,2$ & \\
Campagne & $34 \cdot 51,1$ & \\
Villes & & \\
\hline
\end{tabular}

Source : World Bank, Property alleviation and adjustment in Egypt, volume II, 1990 Korayem K., The impact of economic adjustment policies on the vulnerable families and children in Egypt, a Report prepared for the Third World Forum, Middle East Office and the United Nations Children's Funds (UNICEF), Egypt, 1987. 


\section{NOTES}

1. ANDREA CORNIA Giovanni, «Effects on child welfare » inAdjustment with a human face, a study by UNICEF, éd. Giovanni Andrea Cornia, Richard Holly \& Frances Stewart, Oxford University Press, New-York, 1989, p. 56-59.

2. ANDERSEN Per Pinstrup, JARAMILlO Maurice \& STEWART Frances, "The impact on government expenditure ", in Adjustment with a human face, op. cit. p. 73.

3. Recensement général de la population, de l'habitat et des établissements, année 1986, Central administration for public mobilization and statistics (CAPMAS).

4. Calculé à partir du Rapport de la Banque Centrale 1991.

5. ABD AL-KHALIQ G., Recent and future stabilization experience in Egypt, prepared for the UNU WIDER on stabilization and adjustment programs and policies in developing countries and the role of international institutions, SAPP, 1986.

6. Foreign economic trends and their implications for the United States, Report for the Arab Republic of Egypt, the United States Embassy, Cairo, June 1992.

7. Les évolutions relatées par l'auteur concernent une période antérieure à l'application du programme d'ajustement structurel contrôlé par le FMI et la Banque mondiale. Si certaines mesures de libéralisation économique mises en œuvre depuis 1974 vont dans le sens des politiques préconisées par le FMI, de nombreuses réglementations contraires à ces politiques ont été maintenues ou développées depuis cette date: subventions, bonification du crédit, surévaluation du taux de change, etc. Il est donc difficile de distinguer, parmi les évolutions constatées, ce qui résulte directement des mesures de libéralisation et ce qui découle des politiques interventionnistes (NDLR).

8. PEACOCK Rowley, Welfare economics, Martin Robertson, 1975, p. 13.

9. Poverty alleviation and adjustment in Egypt, document of the World Bank, Report $n^{\circ} 8515-E G I$, Main Report p. 91.

10. World Bank, Arab Republic of Egypt, Country economic memorandum, Economic readjustment with growth, august 30-1989.

11. Poverty alleviation, op. cit. p. 47-51.

12. Ibid.

13. CAPMAS (recensement de 1986, op. cit.).

14. Données du Centre d'information du secteur public concernant la main-d'œuvre dans ce secteur.

15. Calculé à partir des données du Bulletin de production industrielle 1987/88, CAPMAS.

16. Poverty alleviation, op. cit. p. 99.

17. NASSAR Sa'ad, La politique des prix agricoles dans le cadre des politiques d'ajustement structurel en Égypte, Ministère de l'Agriculture, Organisation des Nations Unies pour l'Alimentation et l'Agriculture, janvier 1992, p. 1-5.

18. MINIAWY A., A report on agricultural policies in Egypt, Draft for the IFPRI, 1991.

19. KORAYEM K., The Egyptian economy and the poor in the eighties, Main features and the identification of the poor, the Institute of National Planning, ARE, déc. 1991.

20. KENNEDY E., Food security and nutrition in Egypt, USAID Egypt, 1989.

21. Ministère de l'Agriculture (Budgets de l'alimentation).

22. ALDERMAN H. \& VON BRAUN J., The effects of Egyptian food ration and subsidy system on income distribution and consumption, International Food Policy Research Institute, july 1984, tab. 84.235.

23. Impact de l'augmentation des prix alimentaires sur la nutrition des familles, Institut de la Nutrition, Le Caire, 1989, p. 1. 
24. On sait que cette catégorie contribue pour une part importante à la production de biens alimentaires et a sévèrement pâti de la fixation du prix de ses produits, outre le fait qu'elle bénéficie peu de ce système puisqu'elle s'appuie également sur sa production pour satisfaire ses besoins de consommation.

25. Impact de l'augmentation des prix alimentaires..., source citée.

26. KAMEL A., A report on nutrition problems for the International Food Policy Research Institute, the ACC/Scn, Cairo, 1991.

27. INF, Country Report, 1991.

28. ANDERSEN Per Pinstrup, JARAMILLO Maurice \& STEWART Frances, op. cit., p. 77-78.

29. Poverty alleviation..., op. cit., p. 59.

30. Recensement général... 1986, op. cit., p. 62.

31. Poverty alleviation..., op. cit., p. 60.

32. Ibid. p. 60-63.

33. Recensement général... 1986, op. cit.

34. NASSAR Heba, Impact des politiques de libéralisation économique sur la santé en Égypte. Colloque sur la libéralisation économique en Égypte, Centre de recherches et d'études économiques et financières, 1991, p. 8.

35. Ibid.

36. Calculé à partir des données du ministère de la Santé.

37. SIRAG AL-DIN J. \& DIOP, Strategies for Analysis of Health, Survey Data, League of Arab States, Pan Arab Project for Child Development, Tunis, 1990, p. 8-10.

38. Rapport sur le développement des ressources humaines, 1992. Programme des Nations Unies pour le Développement, New York, 1992.

39. Poverty alleviation..., op. cit., p. 118.

40. AZER Adel, RAMZI Nahed, KARIM Ezza, MUSTAFA Ala, Le phénomène du travail des enfants, Centre national de recherches sociales et criminologiques (CNRSC), Organisation des Nations Unies pour l'Enfance, Le Caire, 1991, p. 1.

41. NASSIM Sulayman, TAWFIQ Awad. ABD AL-MALIK Rasmi, ABD AL-HAMID Tal'at, L'égalité des chances dans la politique de l'enseignement en Égypte, CNRSC, Le Caire, 1991, p. 77 et s.

42. En notant les différences de méthodologies dans les deux rapports.

43. KORAYEM K., op. cit. p. 32.

44. En supposant qu'un pourcentage important de retraités n'ait pas d'enfants en âge scolaire.

45. Poverty alleviation..., op. cit., p. 110-111.

46. Rapport sur le développement des ressources humaines 1992, source, citée.

47. HALIM Nadia, MASA Ali, colloque «Vers une prise en charge complémentaire des personnes âgées ", CNRSC, Le Caire, 1991.

48. AID, Cost recovery project for health sector, project $n^{\circ} 263-0170$.

49. HANSEN N. M., "Development from above, The center down development paradigm " in Walter B. Stohr, R. Fraster Taylor, Development from above on below, John Wiley and son, 1981, p. 17.

\section{INDEX}

Mots-clés : économie, politiques d'ajustement 
AUTEUR

HEBA AHMED NASSAR

Université du Caire 